\title{
Combined Tamoxifen and L-Carnitine Therapies for the Treatment of Idiopathic Male Infertility Attending Intracytoplasmic Sperm Injection: A Randomized Controlled Trial
}

\author{
${ }^{1}$ Milat Haje, ${ }^{2}$ Kameel Naoom
}

\begin{abstract}
Aim: The aim of the present study is to evaluate the fertility outcomes of intracytoplasmic sperm injection (ICSI) as well as sperm count, motility and morphology in couples with infertile male partners exhibiting idiopathic oligoasthenozoospermia $(\mathrm{OA})$ and treated with tamoxifen citrate and/or L-carnitine.

Materials and methods: In this randomized controlled trail, couples with female cause of infertility were excluded. Only couples with male cause of infertility with idiopathic OA were admitted to this study and randomly assigned into four different groups of treatments as follow: Group $A(n=45)$ received an anti-estrogen compound (tamoxifen $20 \mathrm{mg} /$ day), group $B$ $(n=20)$ received L-carnitine (1000 mg/day), group $C(n=34)$ received tamoxifen $20 \mathrm{mg} /$ day plus L-carnitine $1000 \mathrm{mg} /$ day, whereas group $D(n=29)$ received placebo. Treatments were continued for 3 to 6 months.
\end{abstract}

Results: Treatment groups of A, B, and C showed an overall improvement in the tested parameters of sperm when compared to the control group that showed an overall reduction in those parameters after termination of the treatment. In this context, sperm count increased from $7.58 \pm 2.93 \times 10^{6} / \mathrm{ml}$ before treatment to $10.81 \pm 1.84 \times 10^{6} / \mathrm{ml}$ after treatment in group $A(p=0.016)$. Similarly, sperm count increased from $5.32 \pm 2.09 \times 10^{6} / \mathrm{ml}$ to $8.92 \pm 2.29 \times 10^{6} / \mathrm{ml}$ in group $C(p=0.01)$. Patients from group $C$ did not only have an improved total motility of sperm from $8.03 \pm 1.59 \%$ to $13.78 \pm 3.85 \%(p=0.045)$ but also an improved sperm normal morphology from $0.88 \pm 0.45 \%$ to $1.99 \pm 0.71 \%$ $(p=0.026)$. Patients from group $A$ or $C$ exhibited an improved ICSI outcomes when compared to those in patients from group $\mathrm{B}$ or $\mathrm{D}$ (48.9 or 48.3 vs 16.6 or 20 , respectively, $\mathrm{p}=0.46$ ).

Conclusion: It is concluded that administration of tamoxifen and L-carnitine can improve both sperm parameters of fertility and ICSI outcomes. Combined tamoxifen and L-carnitine treatments result in maximum therapeutic effect in men with idiopathic OA.

Keywords: Tamoxifen, L-Carnitine, Male infertility, ICSI.

How to cite this article: Haje M, Naoom K. Combined Tamoxifen and L-Carnitine Therapies for the Treatment of Idiopathic Male

${ }^{1}$ Lecturer, ${ }^{2}$ Professor

${ }^{1}$ Department of Anatomy, Hawler Medical University, Erbil Kurdistan, Iraq

${ }^{2}$ Department of Histology, Hawler Medical University, Erbil Kurdistan, Iraq

Corresponding Author: Milat Haje, Lecturer, Department of Anatomy, Hawler Medical University, Erbil, Kurdistan, Iraq Phone: 009647504532862, e-mail: milathaji@yahoo.com
Infertility Attending Intracytoplasmic Sperm Injection: A Randomized Controlled Trial. Int J Infertil Fetal Med 2015;6(1):20-24.

Source of support: Nil.

Conflict of interest: None

Date of Received: 10-01-15

Date of Acceptance: 25-03-15

Date of Publication: April 2015

\section{INTRODUCTION}

Men with idiopathic infertility are prone to receive a number of empirical therapies, such as FSH; androgens, mesterolone and testosterone undecanoate/enanthate; antiestrogen, clomiphene citrate or tamoxifen; antioxidants, glutathione, lycopene, vitamin E; and/or sperm vitilizers, L-carnitine and coQ10. ${ }^{1,2}$

Aromatization of testosterone to estradiol is important in the negative feedback regulation of pituitary gonadotropin secretion. ${ }^{3}$ Blocking of estrogen at the receptor level of the hypothalamus interferes with the normal negative feedback of sex-steroids, resulting in an increase in endogenous gonadotropin production. Two nonsteroidal anti-estrogens, tamoxifen and clomiphene citrate, have been evaluated for the empirical treatment of idiopathic male infertility. Tamoxifen is favored over clomiphene citrate by some clinicians because it is claimed to have a weaker estrogenic effect. ${ }^{4}$ The latter effect can possibly suppress spermatogenesis if these medications are to be prescribed in high doses. Both tamoxifen and clomiphene citrate are usually administered for 3 to 6 months to improve spermatogenesis as each cycle of spermatogenesis lasts approximately 75 days. $^{5}$

L-carnitine is a known component of epididymal secretions and is now available as an over-the-counter nutritional supplement for the treatment of idiopathic male infertility. In human seminal fluid, approximately $50 \%$ of total carnitine exists as acetyl-carnitine. The compound plays a critical role in intracellular energy metabolism as well as spermatozoa membrane stabilization. Carnitine also has an antioxidant effect and can protect sperm from oxidative damage. ${ }^{6}$ However, the use of carnitine supplementation in idiopathic male infertility remains questionable. ${ }^{7}$ 
Combination therapies of tamoxifen and L-carnitine have been suggested to improve pregnancy rates. Several trails were performed to examine the advantages of such combination therapies in improving pregnancy rates with or without assisted reproductive techniques. In a randomized controlled trial by Comhaire et $\mathrm{al}^{8}$ to evaluate the effectiveness of antioxidants in treatment of infertile men, the investigators found that antioxidants are capable of improving sperm parameters of fertility. On the other hand, the combination therapy of tamoxifen and testosterone undecanoate appears to increase the natural conception rate in men with idiopathic oligozoospermia. ${ }^{9}$ The aim of the present study is, therefore, to assess the effect of combined treatments of tamoxifen, as antiestrogen, and L-carnitine, as an antioxidant, on semen parameters of fertility and pregnancy outcome following intracytoplasmic sperm injection (ICSI) procedure in infertile men with idiopathic oligoasthenozoospermia $(\mathrm{OA})$.

\section{MATERIALS AND METHODS}

This study was done prospectively as a randomized controlled study. Hundred twenty eight men; attending Fertility and IVF Center of Maternity Teaching Hospital in Erbil, Iraq, from January 2013 to June 2014, were admitted to this study. Mean age of male partners was $37.54 \pm$ 2.46 while for female partners was $31.3 \pm 5.8$. The mean infertility duration for patients was $4 \pm 2$ year. Inclusion criteria consisted of repeated exhibition of OA without detectable cause (idiopathic OA). Semen volume, sperm count, total sperm motility, normal morphology, and number of pregnancy after ICSI were evaluated. Exclusion criteria included cases with known etiology of leukocytospermia, altered testicular volume of a minimum of $20 \mathrm{ml}$ as depicted by ultrasonography, ${ }^{10}$ varicocele as detected by clinical examination and ultrasonography, abnormal FSH levels, and/or couples with combined male and female factors. Patients underwent a clinical evaluation including history taking, general examination, genital examination for possible causes of infertility, and semen analyses according to WHO 1999.11

Patients were then randomized into four groups; group A received an antiestrogen compound named tamoxifen $20 \mathrm{mg} /$ day $(n=45)$, group B received an antioxidant named L-carnitine $1000 \mathrm{mg} /$ day $(\mathrm{n}=20)$, group C received both tamoxifen $20 \mathrm{mg}$ /day plus L-carnitine 1000 $\mathrm{mg} /$ day $(\mathrm{n}=34)$, and group D received placebo $(\mathrm{n}=29)$. Treatment was continued for 3 to 6 months followed by ICSI. Semen analysis was performed at least twice before commencing the treatments and twice (1 month apart) at the end of treatments. The incidence of pregnancy after ICSI and the main semen parameters were considered as primary and secondary outcomes to measure the effectiveness of treatments, respectively. Flow chart 1 demonstrates the patient flow chart that was followed in this study.

This study was approved by the local committee of the College of Medicine-Hawler Medical University and funded by Hawler Medical University. All Patients signed informed consents, which explained the nature of this study.

\section{RESULTS}

After 3 to 6 months of treatments of men with idiopathic $\mathrm{OA}$, administration of tamoxifen in group A improved mean sperm count significantly from $7.58 \pm 2.93 \times 10^{6} / \mathrm{ml}$ to $10.81 \pm 1.84 \times 10^{6} / \mathrm{ml}(\mathrm{p}=0.016$, Table 1$)$. Administration of combined therapies of tamoxifen and L-carnitine in group $C$ also improved the mean of sperm count form $5.32 \pm 2.09 \times 10^{6} / \mathrm{ml}$ to $8.92 \pm 2.29 \times 10^{6} / \mathrm{ml}(\mathrm{p}=0.01)$. However, administration of L-carnitine in group B did not improve the mean of sperm count significantly $(4.42 \pm$ $1.78 \times 10^{6} / \mathrm{ml}$ vs $\left.6.17 \pm 2.22 \times 10^{6} / \mathrm{ml}, \mathrm{p}=0.123\right)$. In contrast to treatment groups, placebo recipients (group D) showed reduced mean of sperm counts from $10.63 \pm 2.74 \times 10^{6} / \mathrm{ml}$ to $8.2 \pm 2.69 \times 10^{6} / \mathrm{ml}(\mathrm{p}=0.067)$. Total motility of sperms also increased from $8.03 \pm 1.59 \%$ to $13.78 \pm 3.85 \%$ in group $C(p=0.045)$; however, that increase was not significant in treatment groups A and B. Conversely, control group showed a mean percent reduction in sperm motility from $16.37 \pm 4.85$ before treatment to $14.93 \pm 4.96$ at the end of treatment period $(p=0.27)$. Moreover, combined treatment of tamoxifen and L-carnitine also improved normal sperm morphology. In this context, patients from group $C$ showed improvement in sperm normal morphology percent from $0.88 \pm 0.45$ before the start of combination treatment to $1.99 \pm 0.71$ at the end of treatment period $(p=0.026)$. Administration of tamoxifen (group A) or L-carnitine (group B) also improved normal sperm morphology percent from $1.98 \pm 0.23$ to $2.54 \pm 0.8$ and $1.52 \pm 0.19$ to $3.53 \pm 0.25$, but these improvements were not significant, $\mathrm{p}=0.25$ and 0.14 , respectively. Control patients (group D) showed declined rates in normal sperm morphology from $2.32 \pm 1.32$ to $1.39 \pm 0.28$ at the end of treatment period $(p=0.065)$. No remarkable changes in semen volumes were observed along different groups (Table 1).

The highest pregnancy rates were observed in groups A $(48.9 \%)$ and C $(48.3 \%)$ whereas lowest pregnancy rates were observed in groups D (20\%) and B (16.6). Although both treatment protocols of group A and $C$ had markedly improved the pregnancy rate, this therapeutic improvement was statistically insignificant 
Flow chart 1: The patient flow chart (experimental design) for the study

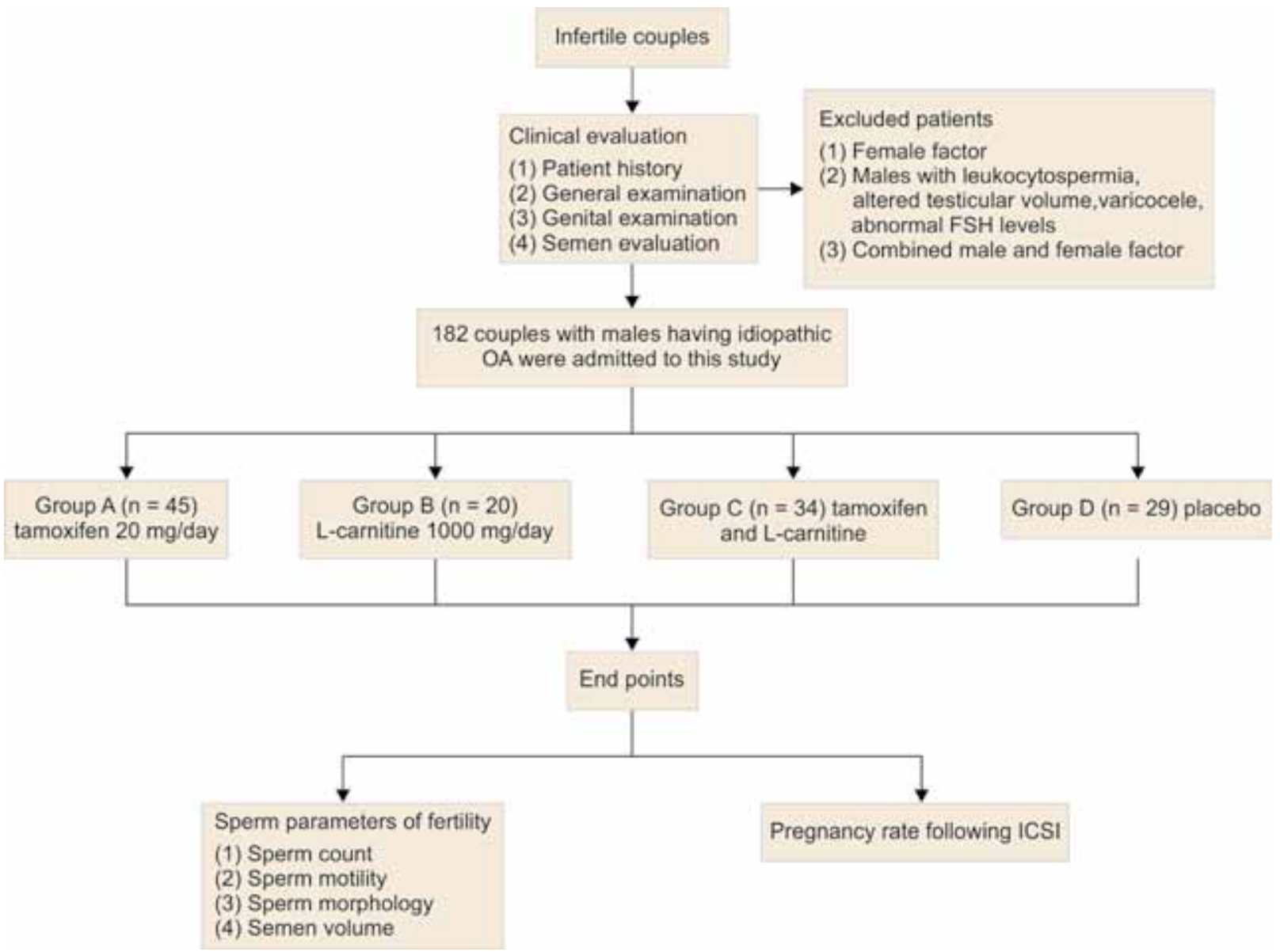

$(\mathrm{p}=0.466)$ using Pearson chi-square analysis (Fig. 1$)$. The overall pregnancy rate following ICSI was $40 \%$ in all treatment groups.

\section{DISCUSSION}

Although hypothalamo-pitutary regulation of testicular and ovarian functions has similarities, the pathology of male and female infertility, and their susceptibility to hormonal therapy, differs markedly. For instance, the predominant mechanism underlying female reproductive dysfunctions is hypothalamic, arising from psychological or metabolic stresses, whereas male reproductive dysfunctions are predominantly testicular, arising from direct genetic or environmental damage. As a result, most of the available treatments are largely ineffective in male reproductive dysfunction. ${ }^{12}$ Moreover, many infertile men receive a description of idiopathic OA rather than a diagnosis because most of pathophysiological mechanisms of male infertility are poorly understood. Furthermore, hormonal treatments are not feasible in male infertility, and they have no established roles in empirical therapies. This suggests that hormonal regulation of spermatogenesis and sperm maturation and function is complex. In addition, although the role of hormonal therapies in male infertility is limited, hormonal therapies are important and effective treatments in cases of gonadotropin deficiency. ${ }^{12}$

Table 1: Effect of different types of treatments on sperm parameters

\begin{tabular}{|c|c|c|c|c|c|c|c|c|}
\hline \multirow[b]{2}{*}{ Groups } & \multicolumn{4}{|c|}{ Semen parameters before treatment } & \multicolumn{4}{|c|}{ Semen parameters after treatment } \\
\hline & Volume $(m l)$ & Count $\left(\times 10^{6} / \mathrm{ml}\right)$ & Total motility (\%) & $\begin{array}{l}\text { Norm. } \\
\text { morph. (\%) }\end{array}$ & Volume (ml) & Count $\left(\times 10^{6} / \mathrm{ml}\right)$ & $\begin{array}{l}\text { Total motility } \\
\text { (\%) }\end{array}$ & $\begin{array}{l}\text { Norm. } \\
\text { morph. (\%) }\end{array}$ \\
\hline$A$ & $2.71 \pm 1.2$ & $7.58 \pm 2.93$ & $19.67 \pm 3.79$ & $1.98 \pm 0.23$ & $2.75 \pm 1.08$ & $10.81 \pm 1.84^{\mathrm{a}}$ & $22 \pm 3.58$ & $2.54 \pm 0.8$ \\
\hline B & $3.17 \pm 0.52$ & $4.42 \pm 1.78$ & $21.67 \pm$ & 1.52 & $2.55 \pm 0.83$ & 6.17 & $23.33 \pm 3.63$ & 3.53 \\
\hline C & $2.7 \pm 1.11$ & $5.32 \pm 2.09$ & $8.03 \pm 1.59$ & 0.88 & $3.15 \pm 1.99$ & $8.92 \pm 2.29^{b}$ & $13.78 \pm 3.85^{\mathrm{c}}$ & $1.99 \pm 0.71^{d}$ \\
\hline D & $2.57 \pm 1.12$ & $10.63 \pm 2.74$ & $16.37 \pm 4.85$ & $2.39 \pm 1.32$ & $2.44 \pm 0.4$ & $8.2 \pm 2.69$ & $14.93 \pm 4.96$ & $1.39 \pm 0.28$ \\
\hline
\end{tabular}

Norm. Morph.: Normal Morphology; Group A: tamoxifen treatment with $20 \mathrm{mg} / \mathrm{ml}$; Group B: L-carnitine treatment with $1000 \mathrm{mg} / \mathrm{day}$; C: combined treatments of tamoxifen of $20 \mathrm{mg} /$ day plus L-carnitine of $1000 \mathrm{mg} /$ day; Group D: placebo treatment (control group). ${ }^{a}$ is significantly where $p=0.016 ;{ }^{b}$ is significantly different where $p=0.01 ;{ }^{c}$ is significantly different where $p=0.045$; and ${ }^{d}$ is significantly different where $p=0.026$ 


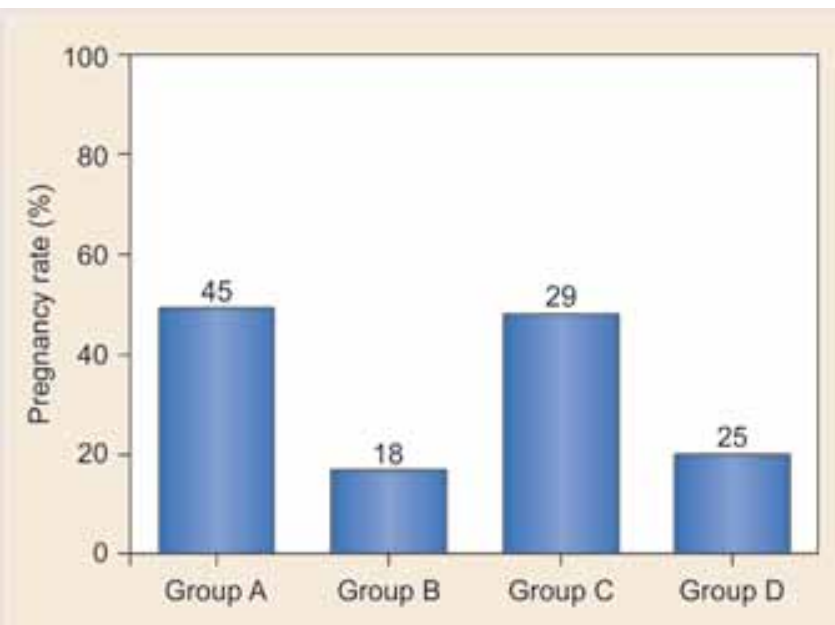

Fig. 1: Pregnancy outcomes following intracytoplasmic sperminjection in men treated with idiopathic oligoasthenozoospermia treated with different medications. Men from group A were treated with tamoxifen $20 \mathrm{mg} / \mathrm{day}$; men from group B were treated with L-carnitine; men from group $C$ were treated with combination therapies of tamoxifen and L-carnitine; and men from group $D$ were treated placebo. Numbers over each bar represent the sample size (n) used. $p=$ 0.466 using Pearson chi-square

This study evaluated the effectiveness of combination of tamoxifen citrate and L-carnitine in the treatment of male infertility. If such combination treatment is confirmed to be effective, such treatment protocol is inexpensive, safe, and easy to administer. Combination therapy for male infertility has been evaluated by other investigators. Adamopolous et $\mathrm{al}^{9}$ studied the therapeutic effectiveness of 6 months of combined tamoxifen and testosterone undecenoate on 212 patients with idiopathic oligozoospermia. That group of investigators showed that the incidence of spontaneous pregnancy was $33.9 \%$ in the active treatment group and $10.3 \%$ in the placebo group. In another study by Ghanem et $\mathrm{al}_{1}^{13}$ most pregnancies occurred between 4 to 6 months of combined clomiphene citrate and vitamin $\mathrm{E}$ treatments. This study also showed a significant improvement in sperm concentration $(p=0.0025)$, forward progressive motility of sperm $(p=0.0286)$, and pregnancy rate. Also sperm concentration improved significantly in combined treatment group receiving tamoxifen and tradamix, which has an androgen-mimetic action. Iacono et $\mathrm{al}^{14}$ also observed an improvement in sperm concentration in tamoxifen recipient patients $(\mathrm{p}=0.002)$. Results of the present study are consistent with the findings of ${ }^{13,14}$ that conclude combination therapies for male infertility is effective and should be further evaluated.

Although married couples are allowed for 1 year of unprotected intercourse to conceive before diagnosed with infertility, many studies allow only an average of 3 months of observation. Results of medical treatment in term of cumulative pregnancy rates may differ if the observation period is long enough $(13,2014)$. In this study, pregnancy rates were not similar in all four groups because improvements in mean percent of count, motility, and normal morphology of sperms only occur in group C. Improvement in mean percent of sperm count also occurs in group A. Although these improvements in sperm parameters of fertility were statistically significant, they did not exceed the fertility cutoff values of more than $20 \times 10^{6}$ sperms $/ \mathrm{ml}$ for sperm count, $40 \%$ for sperm motility percent, and $4 \%$ for normal sperm morphology percent. Moreover, the long duration of infertility may have contributed to the low level of pregnancy rates. In summary, the highest improvements in sperm parameters of fertility and intracytoplasmic sperm injection (ICSI) outcomes were observed in infertile men received combination therapies of tamoxifen and L-carnitine. We, therefore, suggest that combined administration of tamoxifen and L-carnitine in infertile men diagnosed with idiopathic OA, for 3 to 6 months, is beneficial for couples undergo ICSI.

\section{CONCLUSION}

The combination therapy of tamoxifen and L-carnitine leads to a significant improvement in semen parameters comparing with single use of tamoxifen citrate or L-carnitine or control treatment.

\section{ACKNOWLEDGMENTS}

The author would like to express their thanks to Professor Dr Hiwa Banna, Assistant Professor Dr Fareed H AbdulAhad, and Assistant Professor Dr Ali Dabbagh.

\section{REFERENCES}

1. Sigman M, Jarow JP. Male infertility. In: Walsh PC, Retic AB, Vaughan ED. Jr, Wein AJ (eds Campbell's Urology. 8th ed. Philadelphia, WB Saunders Co, 2002.

2. Vandekerckhove P, Liford R, Vail A, Hughes E. Androgen versus placebo or no treatment for idiopathic oligo/asthenozoospermia. Cochrane database sys review 2007.

3. Hayes FJ, Seminara SB, DeCruz S, Boepple PA, Crowley WF. Aromatase inhibition in the human male reveals a hypothalamic site of estrogen feedback. J Clin Endocrinol Metab 2000;85(9):3027-3035.

4. Devi P. Aniestrogens. Pharmacol Ther 1981;12(3):439-447.

5. Heller CG, Rowley MJ, Heller GV. Clomiphene citrate: a correlation of its effect on sperm concentration and morphology, total gonadotropins, ICSH, estrogen and testosterone excretion, and testicular cytology in normal men. J Clin Endocrinol Metab 1969;29(5):638-649.

6. Agarwal A, Said TM. Carntintines and male infertility. Reprod Biomed online 2004;8(4):376-384.

7. Coccuzza M, Agarwal A. Nonsurgical treatment of male infertility: specific and impiric therapy. Biologics Targets and Therapy 2007;1(3):259-269. 
8. Comhaire F, El Garem Y, Mahmoud A, Eertmans F, Schoonjans F. Combined conventional antioxidant astaxanthin treatment for male infertility: a double blind, randomized trial. Asian J Androl 2005;7(3):257-262.

9. Adamopolous DA, Pappa A, Billa E, Koukkou E, Nicopoulou S, et al. Effectiveness of combined tamoxifen citrate and testosterone undecanoate treatment in men with idiopathic oligozoospermia. Fertil Steril 2003;80(4):914-920.

10. World Health Organization (WHO). WHO laboratory manual for the examination of human semen and sperm-cervical mucus interaction. Ann 1st Super Sanita 2001;37(1):1-123.

11. World Health Organization (WHO). WHO laboratory manual for the examination of human semen and sperm-cervical mucus interaction. 4th Cambridge, University Press, Cambridge 1999.

12. Liu PY, Handelsman DJ. The present and future state of hormonal treatment for male infertility. Hum Reprod Update 2003;9(1):9-23.

13. Ghanem $\mathrm{H}$, Shaeer O, El-Segini A. Combination clomiphene citrate and antioxidant therapy for idiopathic male infertility: a randomized controlled trial. Fertil Steril 2010;93(7):2232-2235.

14. Iacono F, Prezioso D, Ruffo A, Di Lauro G, Illiano E, Romeo G, Romis L. Treating idiopathic male infertility with a combination of tamoxifen citrate and a natural compost with antioxidant and androgen-mimetic action. J Steroids Hormons Sci; open access 2013;1-6. 